\title{
Polynomials Approximation Method for Solving Parabolic Partial Differential Equations
}

\section{Sunday Babuba*}

Federal University Dutse, Ibrahim Aliyu Bye-Pass, P.M.B. 7156, Dutse, Jigawa State - Nigeria

\begin{abstract}
A new numerical method based on the approximation of polynomials is here proposed for solving the one dimensional parabolic partial differential equation arising from unsteady state flow of heat subject to initial and boundary conditions. The method results from discretization of the parabolic partial differential equation which leads to the production of a system of algebraic equations. By solving the system of algebraic equations we obtain the problem approximate solutions.
\end{abstract}

Keywords: Polynomials; Interpolation; Collocation; Parabolic

\section{Introduction}

The development of continuous numerical techniques for solving parabolic partial differential equations in physics and mathematics subject to initial and boundary conditions is a subject of considerable interest. In this paper, we develop a new numerical method based on interpolation and collocation of the equation arising from approximating the polynomials [1-27]. To do this, we let $U(x, t)$ represent the temperature at any point in a rod, whose distance from one end is $x$ and $X$ is the length of the rod. Heat is flowing from $\partial U_{\text {ene }}$ end to another under the influence of the temperature gradient $\partial x$. To make a balance of the rate of heat flow in and out of the element, we consider $R$ for thermal conductivity and $c$ the heat capacity which we assume constants, and $\rho$ the density and $t$ represent the time coordinate. In this study we try to find solution to the heat flow equation:

$$
-R A \frac{\partial U}{d x}-\left(-R A\left(\frac{\partial U}{d x}+\frac{\partial}{\partial x}\left(\frac{\partial U}{\partial x}\right) d x\right)\right)=c \rho(A d x) \frac{\partial U}{\partial t}
$$

Where $A$ is the cross section of the rod and the initial and boundary conditions are given by:

$$
U(x, t)=U(x, 0)=f(x) \text { and } U(0, t)=c_{1}, U(X, 0)=c_{2} \text {, where } c_{1}, c_{2}
$$
are constants.

\section{The Solution Method}

To set up the solution method we select an integer $N$ such that $N>0$. We subdivide the interval $0 \leq x X$ into $N$ equal subintervals with meshes given by $t_{j}=j k, j=\frac{1}{\alpha}\left(\frac{1}{\alpha}\right) M$ where $N h=X$. Similarly, we reverse the roles of $x$ and $t$ and we select an integer $M$ such that $M>0$. We also subdivide the interval $0 \leq t \leq T$ into $M$ equal subintervals with meshes given by $t_{j}=j k, \quad j=\frac{1}{\alpha}\left(\frac{1}{\alpha}\right) M$, where $M k=T$, and $h, k$ are the mesh sizes along space and time coordinates. We seek for the approximate solution $\bar{U}(x, t)$ to $U(x, t)$ of the form:

$$
\begin{aligned}
& \bar{U}(x, t)=\sum_{r=0}^{p-1} a_{r}\left[q_{r}(x, t)+s_{r}(x, t)+\psi_{r}(x, t)+\Phi_{r}(x, t)\right], \\
& x \in\left[x_{i}, x_{i+h}\right], t \in\left[t_{j}, t_{j+k}\right]
\end{aligned}
$$

over $h>0, k>0$ successive subintervals $\left[x_{i}, x_{i+h}\right],\left[t_{j}, t_{j+k}\right]$, such that $0=x_{0}<\ldots<x_{i}<\ldots<x_{N}, 0=t_{0}<\ldots<t_{j}<\ldots<t_{M} . p$ is the sum of interpolation points along the space and time coordinates respectively. That is $p=g+b$, where $g$ is the number of interpolation points along the space coordinate, while $b$ is the number of interpolation points along the time coordinate. The bases functions $q_{r}, s_{r} \psi_{r}, \Phi_{r}$, $r=0,1, \ldots, p-1$ are the Taylor, Legendre, chebyshev and Laguerre polynomials which are known, $a_{r}$ are the constants to be determined. The interpolation values $\bar{U}_{i, j}, \ldots, \bar{U}_{i+h-1, j}$ are assumed to have been determined from previous steps, while the method seeks to obtain $\bar{U}_{i+h, j}$ [5-26]. Expanding eqn. (2) we obtain:

$$
\begin{aligned}
& \bar{U}_{i+h, j+k}(x, t)=a_{0}\left(q_{0}+s_{0}+\psi_{0}+\Phi_{0}\right)\left(x_{i+h}, t_{j+k}\right)+a_{1}\left(q_{1}+s_{1}+\psi_{1}+\Phi_{1}\right)\left(x_{i+h}, t_{j+k}\right)+. . \\
& +a_{p-1}\left(q_{p-1}+s_{p-1}+\psi_{p-1}+\Phi_{p-1}\right)\left(x_{i+h}, t_{j+k}\right)
\end{aligned}
$$

We let $h=-\frac{1}{\beta}\left(\frac{1}{\beta}\right)\left[g-\left(\frac{2 \beta-1}{\beta}\right)\right]$, where $g$ is the number of interpolation points along the space coordinate. Then, by Cramer's rule we obtain from eqn. (3),

$$
\begin{aligned}
& W \underline{a}=\underline{F}, \quad \underline{F}=\left(U_{v, j+k}, U_{v+\frac{1}{\beta}, j+k}, \ldots, U_{z, j+k}\right)^{T}, \\
& \underline{a}=\left(a_{0}, \ldots, a_{p-1}\right)^{T} \text { and, }
\end{aligned}
$$

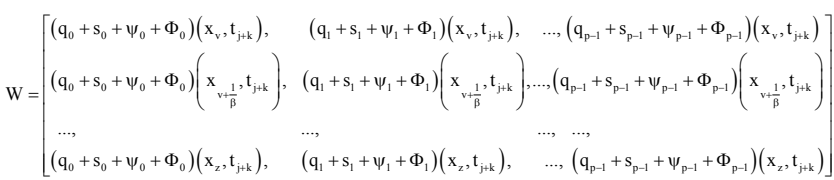

$$
\begin{aligned}
& \text { Where } z=i+g-\left(\frac{2 \beta-1}{\beta}\right), v=i-\frac{1}{\beta} \text { and } W^{-1} \text { exist [10-27]. }
\end{aligned}
$$

Hence, by eqn. (4), we obtain:

$$
\underline{a}=\varpi \underline{F}, \quad \varpi=W^{-1} .
$$

The vector $\underline{a}=\left(a_{0}, \ldots, a_{p-1}\right)^{T}$ is now determined in terms of known

*Corresponding author: Sunday Babuba, Federal University Dutse, Ibrahim Aliyu Bye-Pass, P.M.B. 7156, Dutse, Jigawa State - Nigeria, Tel: +2348039282881; E-mail: sundaydzupu@yahoo.com

Received June 12, 2017; Accepted July 26, 2017; Published July 30, 2017

Citation: Babuba S (2017) Polynomials Approximation Method for Solving Parabolic Partial Differential Equations. J Generalized Lie Theory Appl 11: 277. doi: $10.4172 / 1736-4337.1000277$

Copyright: $\odot 2017$ Babuba S. This is an open-access article distributed under the terms of the Creative Commons Attribution License, which permits unrestricted use, distribution, and reproduction in any medium, provided the original author and source are credited. 
parameters in $\varpi \underline{F}$. If $\varpi_{r+1}, r=0, \ldots, p-1$ is the $(r+1)^{\text {th }}$ row of $\omega$, then,

$$
a_{r}=\underline{\varpi}_{r+1} \underline{F}
$$

Eqn. (6) determines the values of $a_{r}, r=0,1, \ldots, p-1$.

We take first and second derivatives of eqn. (2) with respect to $x$,

$$
\begin{aligned}
& \bar{U}^{\prime}(x, t)=\sum_{r=0}^{p-1}\left[a_{r}\left(q_{r}^{\prime}(x, t)+s_{r}^{\prime}(x, t)+\psi_{r}^{\prime}(x, t)+\Phi_{r}^{\prime}(x, t)\right)\right] \\
& \bar{U}^{\prime \prime}(x, t)=\sum_{r=0}^{p-1}\left[a_{r}\left(q_{r}{ }^{\prime \prime}(x, t)+s_{r}{ }^{\prime \prime}(x, t)+\psi_{r}^{\prime \prime}(x, t)+\Phi_{r}{ }^{\prime \prime}(x, t)\right)\right]
\end{aligned}
$$

Putting eqn. (6) in eqn. (7) we obtain:

$$
\bar{U}^{\prime \prime}(x, t)=\sum_{r=0}^{p-1}\left[\underline{\varpi}_{r+1} \underline{F}\left(q_{r}^{\prime \prime}(x, t)+s_{r}^{\prime \prime}(x, t)+\psi_{r}^{\prime \prime}(x, t)+\Phi_{r}^{\prime \prime}(x, t)\right)\right]
$$

We reverse the roles of $x$ and $t$ in eqn. (3) and we set $k=0\left(\frac{1}{\alpha}\right)\left[b-\left(\frac{\alpha-1}{\alpha}\right)\right]$, where $b$ is the number of interpolation points along the time coordinate, then by Cramer's rule we obtain,

$$
\begin{aligned}
& Y \underline{a}=\underline{E}, \quad \underline{E}=\left(U_{i+h, \eta-\frac{1}{\alpha}}, U_{i+h, \eta}, \ldots, U_{i+h, \gamma}\right)^{T} \\
& \underline{a}=\left(a_{0}, \ldots, a_{p-1}\right)^{T} \text { and, }
\end{aligned}
$$

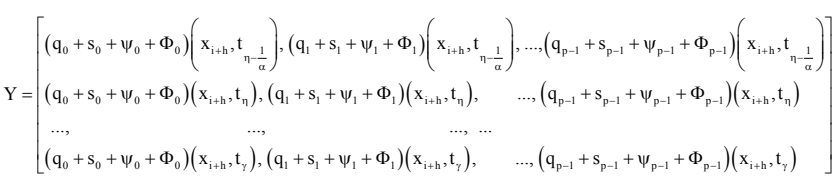

$$
\begin{aligned}
& \text { where } \eta=j+\frac{1}{\alpha}, \quad \gamma=j+b-\left(\frac{\alpha-1}{\alpha}\right) \text { and } Y^{-1} \text { exist [1-16]. }
\end{aligned}
$$

Hence, from eqn. (9) we obtain:

$$
\underline{a}=L \underline{E}, \quad L=Y^{-1} \text {. }
$$

The vector $\underline{a}=\left(a_{0}, \ldots, a_{p-2}\right)^{T}$ is now determined in terms of known parameters in $\underline{L} \underline{E}$.

$$
\text { If } L_{r+1}, r=0, \ldots, p-1 \text { is the }(r+1)^{t h} \text { row of } L \text { then, }
$$$$
a_{r}=\underline{L}_{r+1} \underline{E}
$$

Also, eqn. (11) determines the values of $a_{r}, r=0,1, \ldots, p-1$.

Taking the first derivatives of eqn. (2) with respect to $t$ we obtain:

$$
\bar{U}^{\prime}(x, t)=\sum_{r=0}^{p-1}\left[a_{r}\left(q_{r}^{\prime}(x, t)+s_{r}^{\prime}(x, t)+\psi_{r}^{\prime}(x, t)+\Phi_{r}^{\prime}(x, t)\right)\right]
$$

Putting eqn. (11) in eqn. (12) we obtain:

$$
\bar{U}^{\prime}(x, t)=\sum_{r=0}^{p-1}\left[\underline{L}_{r+1} \underline{E}\left(q_{r}^{\prime}(x, t)+s_{r}^{\prime}(x, t)+\psi_{r}^{\prime}(x, t)+\Phi_{r}^{\prime}(x, t)\right)\right]
$$

But by eqn. (1), it is obvious that eqn. (13) is equal to eqn. (8), therefore,

$$
\begin{aligned}
& \sum_{r=0}^{p-1}\left[\underline{L}_{r+1} \underline{E}\left(q_{r}^{\prime}(x, t)+s_{r}^{\prime}(x, t)+\psi_{r}^{\prime}(x, t)+\Phi_{r}^{\prime}(x, t)\right)\right] \\
& -\sum_{r=0}^{p-1}\left[\underline{\varpi}_{r+1} \underline{F}\left(q_{r}^{\prime \prime}(x, t)+s_{r}^{\prime \prime}(x, t)+\psi_{r}^{\prime \prime}(x, t)+\Phi_{r}^{\prime \prime}(x, t)\right)\right]=0
\end{aligned}
$$

If we collocate eqn. (14) at $x=x_{i}$ and $t=t_{j}$ we obtain a new numerical scheme that solves eqn. (1) explicitly.

\section{Numerical Examples}

In this section, we will test the numerical accuracy of the new method by using the new scheme to solve four (4) test examples. That is, we compute an approximate solution of eqn. (1) at each time level. To achieve this, we truncate the polynomials after second degree. We now specifically use the resultant scheme to solve examples 4.1, 4.2, 4.3 and 4.4 below.

\section{Example 4.1}

Solve for the temperatures in a copper rod $1 \mathrm{~cm}$ long, with the outer curved surface insulated so that heat flows in only one direction. If the initial temperature $\left({ }^{\circ} \mathrm{C}\right)$ within the rod is given as a function of the distance from one face by the equation $U(x, 0)=100 \sin \left(\frac{\pi x}{2}\right)$. Find the temperatures as a function of $x$ and $t$ if both faces are maintained at $\left({ }^{\circ} \mathrm{C}\right)$. Copper has a thermal conductivity $A=0.13 \mathrm{cal} / \mathrm{sec} . \mathrm{cm} .{ }^{\circ} \mathrm{C}$, its heat capacity $c=0.11$ cal.g. ${ }^{\circ} \mathrm{C}$ and density $\rho=7.8 \mathrm{~g} / \mathrm{cm}^{3}$

To solve example 3.1, therefore, we simplify eqn. (1) and apply eqn. (16) to solve it.

By simplification eqn. (1) becomes $A \frac{\partial^{2} U}{\partial x^{2}}=c \rho \frac{\partial U}{\partial t}$. To solve this equation, we take $\Delta x=0.25 \mathrm{~cm}$, then we find $\Delta t$ by the relation $\frac{k \Delta t}{c p(\Delta x)^{2}}=4 \Rightarrow, \Delta t=28.78 \mathrm{sec}$. we also take $\beta=4, \quad \alpha=128$, it implies that $v=i-\frac{1}{4}, z=i+\frac{1}{4}$ and $\eta=\gamma=j+\frac{1}{128}$. We take two interpolation points along space coordinate and one interpolation point along time coordinate. This implies that $g=2, b=1$, and $p=3$. Therefore, for $i=\frac{1}{4}, \frac{1}{2}, \frac{3}{4}, \ldots$, and $j=\frac{1}{128}, \frac{1}{64}, \frac{3}{128}, \ldots$, implies that $h=-\frac{1}{4}, 0, \frac{1}{4}$ and $k=0, \frac{1}{128}$, then the calculated temperatures and concentrations of alcohol are as shown below Table 1 .

\section{Example 4.2}

A hollow tube $20 \mathrm{~cm}$ long is initially filled with air containing $2 \%$ of ethyl alcohol vapors. At the bottom of the tube is a pool of alcohol which evaporates into the stagnant gas above. (Heat transfers to the alcohol from the surroundings to maintain a constant temperature of $30^{\circ} \mathrm{C}$, at which temperature the vapor pressure is $0.1 \mathrm{~atm}$.) At the upper end of the tube, the alcohol vapors dissipate to the outside air, so the concentration is essentially zero. Considering only the effects of molecular diffusion, determine the concentration of alcohol as a function of time and the distance $x$ measured from the top of the tube. Molecular diffusion follows the law:

$$
\frac{\partial c}{\partial t}=D \frac{\partial^{2} c}{\partial x^{2}} \text {, where } \mathrm{D} \text { is the diffusion coefficient, with units of }
$$

$\mathrm{cm}^{2} / \mathrm{sec}$. (This is the same as for the ratio $k / c p$, which is often termed thermal diffusivity.) For ethyl alcohol, $\mathrm{D}=0.102 \mathrm{~cm}^{2} / \mathrm{sec}$ at $30^{\circ} \mathrm{C}$, and the vapor pressure is such that 10 volume percent alcohol in air is present at the surface. Our initial condition is $c(x, 0)=2.0$. The boundary

\begin{tabular}{|c|c|c|c|c|c|c|}
\hline$t$ & $x=0$ & $x=0.25$ & $x=0.50$ & $x=0.75$ & $x=1.00$ & $x=1.25$ \\
\hline 0.00 & 0.00 & 38.27 & 70.71 & 92.39 & 100 & 92.39 \\
\hline 28.78 & 0.0 & 37.54 & 69.37 & 90.63 & 98.10 & 90.63 \\
\hline 57.57 & 0.0 & 36.83 & 68.05 & 88.91 & 96.23 & 88.91 \\
\hline 86.35 & 0.0 & 36.13 & 66.76 & 87.23 & 94.40 & 87.23 \\
\hline 115.51 & 0.0 & 35.45 & 65.49 & 85.59 & 92.61 & 85.59 \\
\hline 143.91 & 0.0 & 34.77 & 64.24 & 83.94 & 90.85 & 83.94 \\
\hline \multicolumn{7}{|c|}{ Table 1: Calculated temperatures. } \\
\hline
\end{tabular}


Citation: Babuba S (2017) Polynomials Approximation Method for Solving Parabolic Partial Differential Equations. J Generalized Lie Theory Appl 11: 277. doi: $10.4172 / 1736-4337.1000277$

Page 3 of 4

conditions are $c(0, t)=0, c(20, t)=10$. To solve this resulting equation we subdivide the length of the tube into five intervals, such that $\Delta x=4 \mathrm{~cm}$. Using the maximum value permitted for $\Delta t$ yields:

$D \frac{\Delta t}{(\Delta x)^{2}}=1, \quad 0.102 \frac{\Delta t}{4^{2}}=1, \Delta t=134.45 \sec s$. We also take $\beta=4$, $\alpha==32$, it implies that $v=i-\frac{1}{4}, z=i+\frac{1}{4}$ and $\eta=\gamma=j+\frac{1}{32}$.

We take two interpolation points along space coordinate and one interpolation point along time coordinate. This implies that $g=2, b=1$, and $p=3$. For $i=\frac{1}{4}, \frac{1}{2}, \frac{3}{4}, \ldots$, and $j=\frac{1}{32}, \frac{1}{16}, \frac{3}{32}, \ldots$, this implies that $h=-\frac{1}{4}, 0, \frac{1}{4}$ and $k=0, \frac{1}{32}$, then the calculated concentrations of alcohol in Table 2.

\section{Example 4.3}

Use the scheme to approximate the solution to the heat equation (Table 3).

$$
\begin{aligned}
& \frac{\partial U}{\partial t}-\frac{\partial^{2} U}{\partial x^{2}}=0,0<x<1 \quad 0<t \\
& U(0, t)=U(1, t)=0, t>0
\end{aligned}
$$

$$
U(x, 0)=\sin \pi x, \quad 0 \leq x \leq 1
$$

\section{Example 4.4}

Use the scheme to approximate the solution to the heat equation (Table 4).

$$
\begin{array}{ll}
\frac{\partial U}{\partial t}-\frac{\partial^{2} U}{\partial x^{2}}=0 & 0<t \\
U(-1, t)=U(1, t)=0, & t>0 \\
U(x, 0)=\cos \left(\frac{\pi x}{2}\right), & -1 \leq x \leq 1, t=0
\end{array}
$$

\section{Conclusion}

In this work, I proposed a method to find the solution of the

\begin{tabular}{|c|c|c|c|c|c|c|}
\hline$t$ & $x=0$ & $x=4$ & $x=8$ & $x=12$ & $x=16$ & $x=20$ \\
\hline 0.0 & 0.0 & 2 & 2 & 2 & 2 & 10 \\
\hline 134.45 & 0.0 & 1.75 & 2 & 2 & 6 & 10 \\
\hline 268.90 & 0.0 & 1.56 & 1.97 & 3.50 & 7 & 10 \\
\hline 403.35 & 0.0 & 1.42 & 2.11 & 3.75 & 6.94 & 10 \\
\hline 537.80 & 0.0 & 1.33 & 2.23 & 3.94 & 6.92 & 10 \\
\hline 672.25 & 0.0 & 1.28 & 1.67 & 4.10 & 6.93 & 10 \\
\hline
\end{tabular}
system of ordinary differential equations arising from discretizing the parabolic partial differential equations with respect to space variables.

\begin{tabular}{|c|c|c|c|c|c|}
\hline \multirow[t]{2}{*}{$x$} & \multirow[t]{2}{*}{ Computed solution $U(x, t)$} & \multirow[t]{2}{*}{ Exact solution $U(x, t)$} & \multirow[t]{2}{*}{ Schmidt Method $U(x, t)$} & \multicolumn{2}{|c|}{ Errors } \\
\hline & & & & New Method & Schmidt Method \\
\hline 0 & 0 & 0 & 0 & 0 & 0 \\
\hline 0.1 & 0.308008706 & 0.308002141 & 0.307963277 & $6.6 \times E-6$ & $2.1 \times E-4$ \\
\hline 0.2 & 0.585867367 & 0.585854886 & 0.58577788 & $1.2 \times E-5$ & $4.0 \times E-4$ \\
\hline 0.3 & 0.806377253 & 0.806360073 & 0.806254085 & $1.7 \times E-5$ & $5.6 \times \mathrm{E}-4$ \\
\hline 0.4 & 0.947953314 & 0.947932118 & 0.947808521 & $2.0 \times E-5$ & $6.6 \times E-4$ \\
\hline 0.5 & 0.996737101 & 0.996715865 & 0.996584857 & $2.1 \times E-5$ & $1.2 \times E-4$ \\
\hline 0.6 & 0.947953314 & 0.947932118 & 0.947808521 & $2.0 \times E-5$ & $6.6 \times \mathrm{E}-4$ \\
\hline 0.7 & 0.806377253 & 0.806360073 & 0.806254085 & $1.7 \times E-5$ & $5.6 \times \mathrm{E}-4$ \\
\hline 0.8 & 0.585867367 & 0.585854886 & 0.58577788 & $1.2 \times E-5$ & $4.0 \times E-4$ \\
\hline 0.9 & 0.398221058 & 0.308002141 & 0.307963277 & $6.6 \times \mathrm{E}-6$ & $2.1 \times E-4$ \\
\hline 1.0 & 0 & 0 & 0 & 0 & 0 \\
\hline
\end{tabular}
Discretization of many of the partial differential equations result in this type of the systems and the proposed method can be applied for

\begin{tabular}{|c|c|c|c|c|c|}
\hline \multirow[t]{2}{*}{$x$} & \multirow[t]{2}{*}{ Exact Solution $U(x, t)$} & \multirow[t]{2}{*}{ Computed Solution $U(x, t)$} & \multirow[t]{2}{*}{ Schmidt method } & \multicolumn{2}{|c|}{ Errors } \\
\hline & & & & New Method & Schmidt Method \\
\hline-1.0 & 0 & 0 & 0 & 0 & 0 \\
\hline-0.75 & 0.380721639 & 0.380741429 & 0.380659316 & $1.9 \times \mathrm{E}-5$ & $4.2 \times E-4$ \\
\hline-0.50 & 0.703481860 & 0.703518427 & 0.703366704 & $3.7 \times \mathrm{E}-5$ & $7.9 \times E-4$ \\
\hline-0.25 & 0.919143346 & 0.919191122 & 0.918992885 & $4.8 \times E-5$ & $1.0 \times E-3$ \\
\hline 0 & 0.994873588 & 0.994925302 & 0.995899602 & $5.2 \times E-5$ & $2.3 \times E-3$ \\
\hline 0.25 & 0.919143346 & 0.911191122 & 0.918992885 & $4.8 \times \mathrm{E}-5$ & $1.0 \times \mathrm{E}-3$ \\
\hline 0.50 & 0.703481860 & 0.703518427 & 0.703366704 & $3.7 \times E-5$ & $7.9 \times E-4$ \\
\hline 0.75 & 0.380721639 & 0.380741429 & 0.380659316 & $1.9 \times \mathrm{E}-5$ & $4.2 \times E-4$ \\
\hline 1.00 & 0 & 0 & 0 & 0 & 0 \\
\hline
\end{tabular}
computation the solutions of the systems.

Table 2: Calculated concentrations of alcohol.

Table 3: Result of action of the scheme on problem.

Table 4: Result of action of the new scheme on problem. 
Citation: Babuba S (2017) Polynomials Approximation Method for Solving Parabolic Partial Differential Equations. J Generalized Lie Theory Appl 11: 277. doi: $10.4172 / 1736-4337.1000277$

Page 4 of 4

\section{References}

1. Adam A, David R (2002) One dimensional heat equation.

2. Awoyemi DO (2002) An Algorithmic collocation approach for direct solution of special fourth - order initial value problems of ordinary differential equations. Journal of the Nigerian Association of Mathematical Physics 6: 271-284.

3. Awoyemi DO (2003) A p-stable linear multistep method for solving general third order Ordinary differential equations. Int J Computer Math 80(8): 987-993.

4. Bao W, Jaksch P, Markowich PA (2003) Numerical solution of the Gross Pitaevskii equation for Bose - Einstein condensation. J Compt Phys 187(1): 18-342.

5. Benner P, Mena H (2004) BDF methods for large scale differential Riccati equations in proc. of mathematical theory of network and systems. MTNS. Edited by Moore BD, Motmans B, Willems J, Dooren PV, Blondel V.

6. Bensoussan A, Da Prato G, Delfour M, Mitter S (2007) Representation and control of infinite dimensional systems (2ndedn), Birkhauser: Boston MA, Motmans B, Willems J, Dooren PV, Blondel V.

7. Biazar J, Ebrahimi H (2005) An approximation to the solution of hyperbolic equation By a domain decomposition method and comparison with characteristics Methods. Appl Math and Comput 163: 633-648.

8. Brown PLT (1979) A transient heat conduction problem. AICHE Journal 16: 207-215.

9. Chawla MM, Katti CP (1979) Finite difference methods for two - point boundary value problems involving high - order differential equations. BIT 19(1): 27-33.

10. Cook RD (1974) Concepts and Application of Finite Element Analysis: NY Wiley Eastern Limited.

11. Crandall SH (1955) An optimum implicit recurrence formula for the heat conduction equation. Quarterly of Applied Mathematics 13(3): 318-320.

12. Crane RL, Klopfenstein RW (1965) A predictor - corrector algorithm with increased range of absolute stability. JACM 12(2): 227-241.

13. Crank J, Nicolson P (1947) A practical method for numerical evaluation of solutions of partial differential equations of heat conduction type. Proc Camb Phil Soc 6: 32-50.

14. Dahlquist G, Bjorck A (1974) Numerical methods. NY: Prentice Hall.

15. Dehghan M (2003) Numerical solution of a parabolic equation with non - local boundary specification. Appl Math Comput 145: 185-194.

16. Dieci L (1992) Numerical analysis. SIAM Journal 29(3): 781-815.

17. Douglas J (1961) A Survey of Numerical Methods for Parabolic Differentia Equations in advances in computer II. Academic press.

18. D' Yakonov YeG (1963) On the application of disintegrating difference operators. Z Vycist Mat I Mat Fiz 3(2): 385-388.

19. Weideman JAC (2010) Computation of the matrix exponential with application to linear parabolic PDEs.

20. Fox $L$ (1962) Numerical Solution of Ordinary and Partial Differential Equation New York: Pergamon.

21. Penzl T (2000) Matrix analysis. SIAM J 21: 1401-1418.

22. Pierre $J(2008)$ Numerical solution of the dirichlet problem for elliptic parabolic Equations. SIAM J Soc Indust Appl Math 6(3): 458-466.

23. Richard LB, Albert C (1981) Numerical analysis. Berlin: Prindle, Weber and Schmidt, inc.

24. Richard L, Burden J, Douglas F (2001) Numerical analysis. Seventh ed., Berlin: Thomson Learning Academic Resource Center.

25. Saumaya B, Neela N, Amiya YY (2012) Semi discrete Galerkin method for Equations of Motion arising in Kelvin - Voitght model of viscoelastic fluid flow. Journal of Pure and Applied Science 3: 321-343.

26. Yildiz B, Subasi M (2001) On the optimal control problem for linear Schrodinger equation. Appl Math and Comput 121: 373-381.

27. Zheyin HR, Qiang X (2012) An approximation of incompressible miscible displacement in porous media by mixed finite elements and symmetric finite volume element method of characteristics. Applied Mathematics and Computation, Elsevier 143: 654-672. 